\title{
Primeira transição do cuidado hospitalar para domiciliar da criança com câncer: orientações da equipe multiprofissional
}

First transition from hospital care to home care for children with cancer: guidelines of the multiprofessional team

Primera transición de la atención hospitalaria a la atención domiciliaria de niños con cáncer: directrices del equipo multiprofesional

\author{
Sara Raquel Kuntz ${ }^{1}$ (1) \\ Luiza Maria Gerhardt² (D) \\ Anali Martegani Ferreira ${ }^{1,3}$ (1) \\ Maitê Telles dos Santos ${ }^{1}$ (1) \\ Maria Cristina Flurin Ludwig ${ }^{1}$ (D) \\ Wiliam Wegner ${ }^{1,3}$ (1)
}

1. Hospital de Clínicas de Porto Alegre. Porto Alegre, RS, Brasil.

2. Universidade Federal do Rio Grande do Sul, Escola de enfermagem, Departamento de Enfermagem Médico-Cirúrgico. Porto Alegre, RS, Brasil.

3. Universidade Federal do Rio Grande do Sul, Escola de enfermagem, Departamento de Enfermagem Materno-Infantil. Porto Alegre, RS, Brasil.
Autor correspondente:

Sara Raquel Kuntz.

E-mail: sarasrkuntz@gmail.com.

Recebido em 15/07/2020.

Aprovado em 09/10/2020.

DOI:https://doi.org/10.1590/2177-9465-EAN-2020-0239

\section{Resumo}

Objetivo: Descrever as orientações para a primeira transição do cuidado hospitalar para o domiciliar da criança com câncer sob a ótica da equipe multiprofissional. Método: Estudo de abordagem qualitativa do tipo exploratório-descritivo realizado em um Hospital Universitário do Sul do Brasil, com nove profissionais da equipe multiprofissional de uma unidade de internação de oncologia pediátrica entre julho e setembro de 2018. Foram realizadas entrevistas semiestruturadas submetidas a análise de conteúdo temática. Resultados: Foram identificadas três categorias temáticas: planejamento multiprofissional para a alta hospitalar da criança com câncer e sua família; a equipe multiprofissional frente ao processo de alta hospitalar; orientações para a primeira alta hospitalar a famílias de crianças com câncer recém diagnosticado. Conclusão/implicações para prática: 0 planejamento e sistematização multiprofissional para as orientações da primeira alta hospitalar são fundamentais para contemplar as necessidades do paciente e suas famílias, tendo o enfermeiro papel central. Mostram-se necessárias melhorias nesse processo.

Palavras-chave: Cuidado de transição; Criança; Equipe Multiprofissional; Câncer; Segurança do paciente.

\section{Abstract}

Objective: To describe the guidelines for the first transition from hospital care to home care of children with cancer from the multi-professional team's perspective. Method: A qualitative exploratory-descriptive study conducted in a University Hospital in southern Brazil by nine professionals from the multi-professional team of a pediatric oncology inpatient unit between July and September 2018. Semi-structured interviews were carried out and submitted to thematic content analysis. Results: Three theme categories were identified, namely: multi-professional planning for hospital discharge of children with cancer and their family; the multi-professional team facing the discharge process; guidelines for the first hospital discharge for families of children with newly diagnosed cancer. Conclusion/practical implications: multi-professional planning and systematization for the guidelines of the first hospital discharge are essential to deliberate the needs of the patient and their families, with the nurse having a centra role. Improvements are needed in this process.

Keywords: Transitional care; Child; Multi-professional team; Cancer; Patient safety.

\section{REsumen}

Objetivo: Describir las principales pautas para la primera transición de la atención hospitalaria al contexto domiciliario de niños con cáncer bajo la óptica del equipo multidisciplinario. Método: Estudio cualitativo de tipo exploratorio-descriptivo realizado en un hospital universitario en el sur de Brasil, con nueve profesionales del equipo multiprofesional de una unidad de hospitalización de oncología pediátrica entre julio y septiembre de 2018. Se realizaron entrevistas semiestructuradas que han sido sometidas a análisis de contenido temático. Resultados: Se identificaron tres categorías temáticas: planificación multiprofesional para el alta hospitalaria de niños con cáncer y sus familias; el equipo multidisciplinario frente al proceso de alta hospitalaria; pautas para el primer alta hospitalaria para familias de niños con cáncer recién diagnosticado. Conclusión/implicaciones para la práctica: la planificación y sistematización multiprofesional en relación a las pautas del primer alta hospitalaria son esenciales para contemplar las necesidades del paciente y sus familias, teniendo la enfermera un papel central. Es necesario implementar mejoras en este proceso.

Palabras clave: Cuidado de transición; Niño; Equipo multiprofesional; Cáncer; Seguridad del paciente. 


\section{INTRODUÇÃO}

O diagnóstico de câncer tem um impacto importante na vida da criança e de sua família. O tratamento oncológico é longo e complexo, exige cuidados específicos por uma equipe multiprofissional, e mudanças significativas no dia a dia das famílias envolvidas. Dadas as variedades dos protocolos atualmente utilizados para o tratamento oncológico, o centro de cuidados desviou-se de uma composição exclusivamente hospitalar. Parte do tratamento passa a ser realizado de forma ambulatorial, o que facilita a manutenção da criança no convívio com a família. ${ }^{1,2} \mathrm{~A}$ primeira alta hospitalar tende a ser desafiadora para a família pois esta, muitas vezes, se sente despreparada para assumir sozinha o cuidado da criança no domicílio. ${ }^{3}$

O retorno ao domicílio requer a transição de cuidados, que consiste na movimentação do paciente de um local de cuidado para outro, como por exemplo, do hospital para o domicílio. Essa trajetória, traz vulnerabilidade à segurança do paciente e sua família, em razão de possível inconstância dos cuidados por falta ou perda de informações essenciais. ${ }^{4} \mathrm{~A}$ equipe multiprofissional de saúde tem um papel fundamental para instrumentalizar a família para esta transição do ambiente de cuidado hospitalar para o contexto domiciliar. Os profissionais devem considerar, além dos aspectos clínicos, as necessidades e as experiências do paciente e sua família. ${ }^{3}$

Apesar de muitos resultados favoráveis terem sido imputados à educação do paciente e sua família, dentre os quais se destacam o aumento da adesão ao tratamento, redução do número de internações hospitalares, aperfeiçoamento da autogestão e períodos de estadia hospitalar mais curtos, ${ }^{5}$ não foram identificadas, na literatura nacional consultada, práticas baseadas em evidências de abordagem efetiva para a educação dos pacientes recém diagnosticados e suas famílias. Não foram encontradas pesquisas, no âmbito brasileiro, com foco na atuação da equipe multiprofissional nesse contexto. Os profissionais de saúde usam a própria experiência para definir métodos, tempo e conteúdo das atividades de educação ao paciente e família. ${ }^{6}$

O Children's Oncology Group (COG) Nursing Discipline, reconhecendo a importância da educação dos familiares, identificou como prioridade nas pesquisas a serem realizadas, as relacionadas à compreensão da efetiva prestação de educação para pacientes e famílias em instituições do COG. ${ }^{7}$

A orientação inadequada do paciente e sua família pode influenciar negativamente nos resultados do tratamento. Entender os princípios e estratégias de aprendizagem eficaz para familiares na conjuntura de um diagnóstico de câncer recente na criança é fundamental para que se propicie o bem-estar e a segurança do paciente e da própria família. ${ }^{5}$

Portanto, teve-se como questão norteadora do estudo: Quais são as orientações para a primeira transição do cuidado hospitalar para o domiciliar da criança com câncer sob a ótica da equipe multiprofissional? Para responder a este questionamento, o objetivo geral foi descrever as orientações para a primeira transição do cuidado hospitalar para o domiciliar da criança com câncer, sob a ótica da equipe multiprofissional. Os objetivos específicos foram: conhecer as estratégias que a equipe multiprofissional utiliza no planejamento e orientações para a alta hospitalar da criança recém diagnosticada com câncer e sua família e identificar as orientações da equipe multiprofissional relacionadas à segurança do paciente no ambiente domiciliar.

\section{MÉTODO}

Trata-se de um estudo de abordagem qualitativa do tipo exploratório-descritivo, realizado como Trabalho de Conclusão da Residência (TCR). Foi desenvolvido em uma unidade de internação de oncologia pediátrica de um hospital de referência do Sul do Brasil, no período entre julho e setembro de 2018.

A unidade de internação possui 24 leitos destinados exclusivamente a pacientes onco-hematológicos de 0 a 18 anos. A assistência aos pacientes é oferecida por uma equipe multiprofissional composta por 37 técnicos de enfermagem, 14 enfermeiros, oito médicos, um nutricionista, um psicólogo, um assistente social, um farmacêutico e um educador físico. Atualmente a unidade conta com o suporte de um dentista que concede atendimento aos pacientes em caráter de pesquisa e voluntariado. Também prestam assistência aos pacientes profissionais da Residência Integrada Multiprofissional em Saúde (RIMS) com ênfase em onco-hematologia composta por um enfermeiro, um nutricionista, um farmacêutico, um assistente social e um psicólogo.

O convite para a participação no estudo foi realizado pessoalmente pela própria pesquisadora e posteriormente formalizado por e-mail. Todos os profissionais convidados aceitaram participar.

Integraram o estudo nove profissionais da equipe multiprofissional, sendo dois enfermeiros, dois médicos, um farmacêutico, um nutricionista, um psicólogo, um assistente social e um dentista, selecionados por amostragem intencional, por serem os profissionais responsáveis pelas orientações dos familiares das crianças com câncer hospitalizadas. ${ }^{8}$

Delimitaram-se, como critérios de inclusão, profissionais da equipe multiprofissional que atuam na assistência à criança em tratamento oncológico por no mínimo um ano. Foram excluídos da pesquisa aqueles profissionais afastados do trabalho ou de férias no período da coleta dos dados.

Para a coleta das informações, adotou-se como estratégia a entrevista semiestruturada, conduzida pela própria pesquisadora. Foram elaboradas cincos questões norteadoras que abordavam perguntas sobre como as orientações eram realizadas, quais os profissionais essenciais para esse processo educativo, se existe algum planejamento para o desenvolvimento das orientações, quais orientações têm implicações com a segurança do paciente e se é utilizado algum instrumento para conduzir o processo educativo e as orientações com a criança e a família. Um encontro foi agendado com cada profissional, em sala reservada, com duração aproximada de trinta minutos, e gravado em áudio com a utilização de um gravador digital. As entrevistas foram posteriormente transcritas na íntegra, e o nome do participante foi substituído pela letra "P" seguido do número conforme ordem de 
realização das entrevistas. As falas foram ajustadas do ponto de vista ortográfico para facilitar a compreensão do leitor, porém sem alterar o sentido dado pelo entrevistado. A interrupção da coleta dos dados se deu pelo critério de saturação das informações.

Para a análise das informações, empregou-se a análise de conteúdo do tipo temática proposta por Minayo. ${ }^{9} \mathrm{~A}$ discussão dos resultados foi fundamentada nas literaturas nacional $\mathrm{e}$ internacional relevantes ao tema.

Os profissionais participantes obtiveram feedback dos resultados do estudo através da apresentação pública do mesmo e posteriormente disponibilizado na unidade uma versão impressa.

Os aspectos éticos foram respeitados e seguidos de acordo com a Resolução 466/12 do Conselho Nacional de Saúde. ${ }^{10} \mathrm{O}$ estudo foi aprovado pelo Comitê de Ética em Pesquisa (CEP) do Hospital de Clínicas de Porto Alegre, via Plataforma Brasil, CAAE № 82979618.1.0000.5327. Todos os participantes do estudo assinaram o Termo de Consentimento Livre e Esclarecido (TCLE) aprovado pelo CEP.

\section{RESULTADOS}

Participaram do presente estudo nove profissionais da equipe multiprofissional da oncologia pediátrica com idades que variaram entre 30 a 55 anos de idade. $O$ tempo de atuação na oncologia pediátrica variou de um a 22 anos e o tempo de formado de quatro a 32 anos.

A análise das informações revelou três categorias temáticas: planejamento multiprofissional para a alta hospitalar da criança com câncer e sua família; a equipe multiprofissional frente ao processo de alta hospitalar; orientações para a primeira alta hospitalar a famílias de crianças com câncer recém diagnosticado.

\section{Planejamento multiprofissional para a alta hospitalar da criança com câncer e sua família}

Identificou-se que não há um planejamento multiprofissional estruturado para a realização das orientações e da transição do cuidado hospitalar para o domiciliar, mas sim um processo segmentado e de organização independente de cada profissional. O planejamento conjunto e antecipado foi pontuado como casual, quando identificadas dificuldades da família na compreensão dos cuidados necessários no domicílio.

Apesar de identificarem a importância do planejamento multiprofissional para a alta, alguns entrevistados referiram possuir dificuldades na execução desse processo, como por exemplo quando a alta hospitalar é antecipada ou não prevista. Sentimento de frustração foi mencionado pelos profissionais quando há falhas no processo de orientações para a alta.

\section{[...] na minha opinião não acontece, multiprofissionalmente} não, cada um vai vendo as necessidades daquele paciente e vai planejando de acordo com a sua profissão [...] (P1).

Não acontece um planejamento formal [...]em algumas situações sim, acaba a gente fazendo um planejamento [...] aquele paciente mais grave, ou aquele paciente com uma vulnerabilidade maior, seja ela de compreensão, seja ela social, financeira, enfim, a gente acaba se organizando um pouco melhor para esses casos [...] (P6).

[...] às vezes pela pressa de dar alta o profissional fica frustrado porque não consegue orientar corretamente essa família $[. .].(P 3)$

\section{A equipe multiprofissional frente ao processo de alta hospitalar}

Em relação ao momento da realização das orientações, alguns profissionais relataram aguardar a confirmação da alta, o que por vezes acontece apenas no próprio dia, para iniciar esse processo. Entretanto, quando identificadas dificuldades por parte da família, como insegurança para prestar o cuidado no domicílio, baixa renda, e/ou falta de instrução para ler e escrever (familiares não alfabetizados), dentre outras situações, os participantes referiram buscar antecipar as orientações, de modo a possibilitar melhor aproveitamento, implantando o cuidado de transição. Em contrapartida, há profissionais que sinalizaram iniciar as orientações durante a internação.

[...] a gente vai fazendo o acompanhamento logo após o diagnóstico [...] a partir das demandas [...] com isso a gente já tem uma prévia dos recursos que eles vão precisar para a alta. Então a gente vai trabalhando ao longo do processo de internação, no momento da alta a gente faz um fechamento disso [...]. (P1)

[...] a gente sempre espera a certeza de alta e nisso as vezes fica em cima da hora [...] por exemplo, casos em que famílias não são alfabetizadas [...] a gente tenta começar o processo da alta um pouco antes para que as famílias tenham um pouco mais de segurança na hora de dar alta. (P2)

Os profissionais destacaram, no que se refere às dificuldades para as orientações, que a primeira alta hospitalar é um momento em que os familiares costumam estar muito inseguros pois necessitam incorporar diversas informações em curto período de tempo, ressaltando a importância das ações educativas ocorrerem ao longo de todo o período de internação. Os participantes apontaram acreditar que as orientações fundamentais são fornecidas aos familiares, contudo, há dificuldade para verificar a assimilação das informações.

[...] o que a gente percebe é que existe na primeira internação uma desorganização familiar, a mãe ou o cuidador tem que dar conta das coisas daqui da internação, das coisas que ficaram em casa [...] e a gente vê que eles vão tendo segurança com a equipe no decorrer desta primeira internação [...]. (P9)

[...] a maior dificuldade hoje nem seja tanto fornecer a orientação, mas é garantir que a orientação foi percebida 
[...] acho que às vezes faltam mecanismos para a gente garantir que a familia tem capacidade de implementar [...] de entender o que tu estás dizendo [...]. (P7)

Ao se tratar da responsabilidade pelas orientações, os participantes foram unânimes ao considerar todos os profissionais componentes da equipe multiprofissional como elementares para esse processo. No entanto, indicaram o enfermeiro como referência no que tange à educação da família e do paciente. Porém, ressaltou-se a importância e a necessidade de se realizar o processo de planejamento de alta hospitalar de forma compartilhada.

[...] eu não vejo a farmacêutica distante disso, eu não vejo a assistente social distante disso também, mas eu acho que acaba, em linha de frente, sendo a enfermagem de um modo geral, com relação a isso, a cuidados e educação. (P6)

[...] eu acho que éfundamental que todo mundo trabalhe junto sempre, essa multiprofissionalidade, interdisciplinaridade é essencial, vejo todas as áreas como fundamentais. (P8)

\section{Orientações para a primeira alta hospitalar a famílias de crianças com câncer recém diagnosticado}

Destacaram-se as orientações relacionadas à identificação e reconhecimento de sinais de urgência oncológica, como por exemplo, febre, vômito, diarreia e sangramentos, salientando que tais sinais significam que há necessidade de encaminhamento ao hospital para manejo imediato.

[...] orientação em caso de urgência, o que fazer se o paciente tem sangramento em casa, o que fazer se tiver febre em casa [...] fazer a família entender o que são situações que para a gente são consideradas urgências e que isso significa encaminhamento hospitalar. (P7)

Os participantes também destacaram ser de extrema relevância que as famílias conheçam as diferentes etapas do tratamento oncológico, e que, por meio das orientações oferecidas desenvolvam a compreensão sobre a importância da manutenção dos cuidados destacados. As orientações realizadas pela equipe multiprofissional às famílias precisam ser agregadas ao cotidiano da vida de cada criança. Desse modo, é importante que as famílias tenham confiança na equipe, e esta seja sua referência para esclarecimento de dúvidas que possam surgir tanto no período da internação quanto no que a criança permanece em seu domicílio. Para tanto, é fundamental que sejam disponibilizadas formas para manter contato com os profissionais.

[...] orientações da vida cotidiana desse paciente [...] que vai desde o aspecto de nutrição, de alimentos, da vida em sociedade, se pode ir à escola, se pode ter animal, da casa[...] um paciente, para sair daqui a família tem que ser capaz de saber quais são os sinais de alerta, quando procurar a equipe multiprofissional [...] e como fazer isso [...]. (P7)

[...] sempre falo que a criança que é imunodeprimida, que baixa muito as defesas, em função disso elas se infectam com facilidade então por isso que a gente faz todos esses cuidados aqui, que ela siga esses mesmos cuidados que nós temos aqui, ela faça em casa. (P5)

Quanto às orientações específicas de cada profissional, considerando o paciente pediátrico em tratamento oncológico como uma criança com necessidades especiais de saúde, destacaram-se as relacionadas ao uso de tecnologias de saúde como, por exemplo, o uso de cateter venoso central de longa permanência. Também se enfatizou a alimentação por via enteral, a poli medicação, além do uso de medicamentos de alto risco, como os quimioterápicos, o que se corrobora com as falas subsequentes.

[...] a folhinha amarela que a gente chama, todas as prescrições são colocadas em uma tabela com horários e forma de administração desses medicamentos [...] então a gente orienta sempre cuidados na questão dos horários, a forma de administração e também todo medicamento que precisa realizar uma derivação farmacêutica [...]. (P2)

[...] procuro orientar assim os cuidados com o portocath [...] os cuidados com sonda nasoentérica para quando o paciente vai com sonda [...]. (P3)

Os participantes também trouxeram como aspectos tratados em suas orientações às famílias, a prevenção e o manejo dos sintomas causados pela própria doença ou pelo tratamento quimioterápico, bem como as diferentes formas de obtenção dos medicamentos necessários para a continuidade do tratamento domiciliar, além do acesso à rede de saúde no território dessas famílias.

[...] onde buscar esse medicamento, se esse medicamento tem no posto, se esse medicamento tem no estado, se vai ter que ser comprado, então o acesso a gente também orienta [...]. (P2)

[...] eu reforço muito a importância da higiene bucal para diminuir outras sequelas do tratamento antineoplásico [...] quando eles não conseguirem fazer a higienização com escova por estarem com lesões em boca, por estar doendo, estar enjoado eu ensino a fazer a higiene com gaze[...]. (P8)

Identificaram-se como instrumentos e estratégias utilizados pelos profissionais para as orientações a adaptação da linguagem utilizada de acordo com o nível de compreensão de cada familiar, o uso de materiais educativos impressos, como folders 
institucionais, por exemplo. Citou-se também o emprego de tabelas de medicamentos e a repetição das informações, tanto pelo profissional, quanto pelo familiar, para garantir a assimilação.

[...] procuro falar bem a linguagem delas assim, tem que explicar no entendimento da mãe, a gente não pode explicar com palavras difíceis também. (P3)

[...] temos folders específicos [...] conforme a situação clínica daquele paciente, a gente orienta e entrega mais o material escrito. (P6)

[...] a gente sempre pede para a família reproduzir o que a gente explicou naquele momento, é importante que o receptor da informação repita aquilo [...]. (P2)

Quando identificada dificuldade por parte dos familiares no entendimento e reprodução das informações, criam-se outras estratégias para que esses cuidadores compreendam com mais facilidade. Procuram-se utilizar materiais didáticos demonstrativos e lúdicos para subsidiar a aprendizagem e a compreensão sobre as orientações realizadas às famílias e crianças. Ou seja, utilizam-se diversos materiais disponíveis na unidade para auxiliar o ensino e favorecer a compreensão das informações pelas famílias, tais como, seringas com marcações em cores, bonecos, isto é, maneiras criativas para explicar o cuidado.

[...] quando a gente percebe que não está acontecendo com aquele familiar, a gente procura perguntar se tem alguém que possa vir ajudar, ou quando o paciente é mais adolescente [...] ser envolvido também naquele cuidado [...]. (P6)

[...] já usamos boneca com punção do cateter e com uso da sonda, já instalamos em um bonequinho e mostramos como funcionava, para a criança e para a família uma coisa mais lúdica [...]. (P5)

[...] já teve casos que fez caixinha com cor pra eles, com desenho de comprimidinhos ali, quantos e um reloginho com horário [...]. (P2)

No que se refere à segurança do paciente pediátrico oncológico, no âmbito domiciliar, destacaram-se cuidados relacionados ao armazenamento correto dos medicamentos e à manipulação e descarte de quimioterápicos. Além disso, acentuou-se o cuidado necessário com as possíveis intercorrências que o paciente está sujeito, como febre, diarreia e sangramentos. Situações estas nas quais o paciente deverá ser encaminhado imediatamente ao hospital onde realiza o tratamento ou, quando não for possível, a serviços de saúde próximos do território da família.

[...] todos os pacientes com quimioterápico oral a gente orienta então os cuidados para quem vai administraresses medicamentos [...] trazer para o hospital e descartar no local adequado. (P2)
[...] conseguir minimamente identificar a rede de atendimento naquele território porque se acontece alguma coisa, principalmente pacientes que moram no interior as vezes não dá tempo de chegar [...]. (P1)

Outros aspectos também frisados foram os relacionados às restrições alimentares, ao convívio escolar e à limitação das visitas. Ainda, ressaltou-se o cuidado necessário com a higiene corporal, do ambiente em que vive o paciente e dos alimentos a serem consumidos.

Sem a menor sombra de dúvida, a questão de higiene pessoal e o que a gente chama de higiene alimentar [...] a gente tem trabalhado muito mais essa questão atualmente, de foco na higiene alimentar e do cuidador, do manipulador do alimento, do que propriamente $o$ alimento em si. (P6)

Destacou-se, além do mais, a necessidade de adequar as orientações à realidade socioeconômica da família, de forma que sejam o mais próximo possível do ideal. Outra questão apresentada foi a da imprescindibilidade de um feedback, de saber se as orientações oferecidas ao cuidador foram entendidas adequadamente.

[...] tu tens parâmetros internacionais que te orientam, a casa tem que ter piso, não pode ter cimentos exposto, não pode ter várias coisas assim fumaça, fuligem [...] e aí tu vens para uma realidade social que não é bem isso [...] por mais que se saiba as orientações que são as melhores para o paciente, a realidade social do paciente não permite [...]. (P6)

[...] tu não ter feed back do quanto aquilo foi compreendido pela família, acho que a insegurança não está na ausência de informação, está na ausência de implementação do que foi orientado, corretamente [...]. (P7)

\section{DISCUSSÃO}

Quando uma criança é diagnosticada com câncer, inicia-se um processo no qual ela e sua família experimentam diferentes reações emocionais. Torna-se necessário à família adaptar-se às novas exigências em sua rotina diária, o que impõe alterações na organização e dinâmica familiar, mudanças de comportamentos e aquisição de novos conhecimentos relacionados à doença e seu tratamento para o cuidado seguro da criança, especialmente no domicílio. ${ }^{11,12} \mathrm{~A}$ necessidade de educa-los e habilita-los para essa transição durante a primeira internação é um desafio para os profissionais de saúde. ${ }^{3}$

Os profissionais são responsáveis por identificar as necessidades e vulnerabilidades geradas pelo processo de mudança no cotidiano da família. São responsáveis também por prover conhecimentos, habilitando os membros da família 
para dar continuidade aos cuidados de forma segura, antes realizados no ambiente hospitalar, no domicílio. ${ }^{6,11}$

O desenvolvimento e implementação de um plano de alta é imprescindível para que ocorra a transição segura do paciente para o domicílio. A equipe multiprofissional precisa elaborar esse planejamento de forma a enaltecer a participação da família no tratamento da criança. Planos estruturados de alta hospitalar permitem qualificar os cuidados desde a primeira internação até a manutenção da assistência domiciliar, o que minimiza a ocorrência de hospitalizações não programadas e possibilita que os cuidados domiciliares sejam desenvolvidos de maneira consciente, garantindo a segurança do paciente. ${ }^{13,14}$

Realizar um planejamento prévio para a primeira alta hospitalar possibilita que as orientações sejam feitas de forma sistemática ao longo da hospitalização, evitando excesso de informações em um curto período de tempo, o que poderá prejudicar a assimilação das informações. ${ }^{14-15}$ Contribui também para evitar situações de estresse, sentimento de insegurança para a alta por parte da família, valoriza o trabalho em equipe e a participação da família no tratamento, bem como contribui para continuidade dos cuidados no domicílio.

Identificaram-se lacunas na sistematização do processo de alta, as quais interferem na qualidade das orientações. A falta de um planejamento multidisciplinar para a alta, do cuidado de transição, o qual permitiria uma organização prévia das atividades educativas a serem realizadas desde o início da hospitalização, dificulta a aquisição das informações pela família, o que reduz a percepção de dúvidas, e sequer permite que a equipe avalie o quanto essas orientações foram compreendidas pelos familiares.

O insucesso na prestação ou na implementação das orientações feitas tende a causar sentimento de frustração nos profissionais envolvidos. Este sentimento também foi identificado quando abordado com os familiares, além da sensação de incapacidade de prestar os cuidados à criança, seja pela falha nas orientações por parte dos profissionais ou pela limitação da compressão das informações fornecidas relacionado ao impacto do diagnóstico. ${ }^{3}$

Reconheceu-se, por meio deste estudo, que apesar da importância de todos os profissionais componentes da equipe multiprofissional nas orientações para a alta e na capacitação da família, destaca-se o enfermeiro como profissional de referência nesse processo, o que foi corroborado pela literatura. ${ }^{3,5,13}$

O enfermeiro, como educador, tem a responsabilidade de preparar a família para participação ativa no processo de cuidado à criança. Para tanto, necessita de conhecimento, recursos, tempo e apoio adequados. Todavia, na prática assistencial, por vezes, devido às demandas de atividades e funções que assume (gerenciamento do cuidado, da equipe de enfermagem, e da assistência direta à criança e sua família) pode apresentar dificuldades para desenvolver a dimensão educativa em sua plenitude. A presença de um enfermeiro para atuação exclusiva na educação em saúde na equipe multiprofissional, contribui para o fortalecimento de vínculo entre os membros da equipe assistencial, criança, família e com os serviços que compõem a rede de saúde do território a que a família pertence, favorecendo o retorno seguro para o domicílio. ${ }^{13,16}$

Com o objetivo de aumentar a satisfação da família e dos profissionais da saúde em relação cuidado de transição, por meio de um estudo, desenvolveu-se o programa denominado "Road to Home" (Estrada para Casa). Os resultados de tal pesquisa demonstraram melhorias nos processos de educação, mas também revelaram que os profissionais continuavam com queixas em relação à falta de tempo para efetuar um trabalho satisfatório. A partir disso, foi proposta a inserção de um enfermeiro da equipe para se dirigir principalmente ao ensino dos familiares, o que resultou em mais qualidade nas informações prestadas. ${ }^{15}$

A qualidade dos cuidados prestados pelos familiares à criança no domicílio está diretamente ligada à educação recebida durante a hospitalização. Nesse sentido, identificar as informações primárias que são essenciais para a instrução dos cuidadores, já durante a primeira internação da criança, após o diagnóstico, é fundamental para uma educação padronizada, o que permite assegurar a entrega das informações de forma consistente aos familiares. ${ }^{6,17}$ Ou seja, esclarecer junto à família quais as informações precisam receber para prover cuidados seguros no ambiente doméstico pode contribuir para a educação efetiva. ${ }^{5}$

Neste estudo, destacaram-se orientações elementares a serem fornecidas às famílias durante a primeira internação pós diagnóstico, sobretudo as relacionadas aos sinais de urgência, as medicações e precauções ambientais e alimentares.

Reconhecer os sinais e sintomas comuns de agravamento clínico durante o tratamento oncológico foi salientado pela maioria dos profissionais neste estudo como uma das orientações indispensáveis, bem como em outras pesquisas neste mesmo âmbito, tendo em vista a importância do encaminhamento imediato da criança ao hospital ou ao serviço de referência em seu território. Sendo a febre o sinal mais referido, já que é o principal sinalizador da presença de processos infecciosos, os quais são potencialmente fatais se não tratados precocemente..$^{3,15,18}$

As instruções relacionadas aos cuidados necessários com a higiene do ambiente, do corpo e dos alimentos foram mencionadas pelos profissionais como importante forma de diminuir os riscos de infecções. Porém, sabe-se que a higiene das mãos é a forma mais efetiva e de menor custo para prevenir infecções, ${ }^{19}$ a qual é uma ação realizada pelos profissionais, entretanto, apesar da relevância, essa ação não foi sinalizada como foco nas orientações.

As informações sobre o tratamento medicamentoso, incluindo quimioterápicos, também foram pautadas pelos profissionais participantes deste estudo como instrução fundamental a ser dada aos familiares. Erros na administração de medicamentos, principalmente na dosagem, é um dos problemas mais comuns após a alta e podem interferir diretamente no sucesso do tratamento. Esses problemas podem ser minimizados pelos profissionais da saúde, com a implementação de intervenções demonstrativas quanto à administração dos medicamentos, bem como disponibilização de instruções padronizadas impressas. ${ }^{3,15,18}$ Neste estudo, foi possível identificar estratégias utilizadas por alguns profissionais para qualificar as orientações 
quanto a administração dos medicamentos, como a utilização de seringas marcadas com diferentes cores de acordo com o medicamento e a dose prescrita, bem como a entrega de tabelas com a organização da prescrição médica em uma linguagem acessível aos familiares.

Para uma prática educacional efetiva não basta identificar as informações essenciais, é necessário também qualificar os métodos utilizados para tal. Deve-se levar em consideração as necessidades e particularidades de cada paciente e sua família, no que se refere à capacidade e ao tempo necessário para a compreensão das informações, bem como as condições sociais, econômicas e letramento da família, adequando as recomendações às suas realidades. ${ }^{3,6,20}$

Neste estudo, identificaram-se como instrumentos e métodos, utilizados para a realização das orientações, as práticas lúdicas e demonstrativas, o emprego de materiais impressos, a repetição das informações pelo profissional e principalmente pelo cuidador, e, ainda, a disposição de contato telefônico para o caso de dúvidas. Tais instrumentos e métodos corroboram com as recomendações indicadas para aumentar a qualidade do cuidado e a segurança da criança em tratamento oncológico para a alta hospitalar. ${ }^{4,21}$

Por meio deste estudo, os profissionais participantes demostraram preocupação com a carência de ferramentas para garantir que todas as orientações necessárias sejam fornecidas, bem como mecanismos para identificar o nível de compreensão dos familiares sobre as orientações prestadas antes da primeira alta hospitalar. Cabe à equipe multiprofissional validar a compreensão da família sobre as orientações realizadas. ${ }^{3}$ Para tal, é importante incentivar a família a confirmar com os profissionais a sua compreensão sobre as orientações recebidas, estimulando o familiar a autoquestionar-se, por exemplo, acerca da administração dos medicamentos, os sinais de alerta que indicam piora clínica da criança e sobre os cuidados de prevenção de infecções. ${ }^{21}$

Algumas alternativas estão sendo desenvolvidas para qualificar as práticas educacionais de orientação às famílias de crianças com câncer, com vistas ao cuidado seguro no domicílio. Tem-se como exemplo o desenvolvimento de uma lista de verificação padronizada, que facilita o trabalho da equipe, principalmente dos enfermeiros, na educação das famílias. Tal instrumento é adaptável às necessidades eà realidade econômica e psicossocial de cada família. Esse instrumento faz parte de um programa denominado "Road to Home" anteriormente citado, que é um método interativo de educação entre os profissionais e familiares, composto por uma tábua que simula um jogo de tabuleiro, uma estação de educação que contém materiais escritos e de prática, um manual impresso e um kit com itens necessários para cuidar com segurança da criança no domicílio. ${ }^{15,22}$

Tais alternativas, apesar de terem evidenciado melhorias importantes na qualidade da prática educacional nas instituições participantes dos estudos, necessitam de um investimento financeiro e de aumento do número de profissionais, que não se encaixam na realidade atual das instituições públicas de saúde brasileiras.

\section{CONSIDERAÇÕES FINAIS - IMPLICAÇÕES PARA A PRÁTICA}

O cuidado de transição do hospital para o domicílio, na primeira alta da criança com câncer, é um processo complexo, tanto para os profissionais de saúde quanto, principalmente, para a família. Todos os profissionais envolvidos na assistência a essas crianças e suas famílias são responsáveis por tornar esse processo de transição em um período de aprendizagem, habilitando-os para realizar a continuidade do cuidado seguro no domicílio.

Apesar dessa responsabilidade ser da equipe multiprofissional, evidencia-se o importante papel do enfermeiro nesse processo, pois é o profissional que atua continuamente junto ao paciente, sendo o facilitador na identificação de dificuldades enfrentadas pela família. Reforça-se o papel de educador do enfermeiro na instrumentalização da família da criança com câncer para o cuidado domiciliar, sendo isto uma prioridade no cuidado e que necessita de continuidade, reforço e valorização cotidiana frente às demandas do processo de trabalho.

Evidenciou-se que o conteúdo das orientações prestadas pelos diferentes profissionais corresponde ao necessário para o cuidado seguro no domicílio, corroborando com o encontrado na literatura, porém o processo de planejamento e orientações para a alta precisa ser considerado prioridade, proporcionando que a educação dessas famílias aconteça desde a admissão e que não se esgote nesse primeiro período.

Destacou-se como aspecto essencial no processo de planejamento e execução das orientações para alta, a identificação das necessidades psicossociais de cada criança e suas famílias. Diante disso, sugere-se que, antes de se iniciar o processo de educação, sejam realizadas consultas para acolhimento visando proporcionar escuta qualificada, identificar as demandas de cuidado de cada família, e melhor definir métodos de abordagem.

Além de identificar os conteúdos para a educação das famílias, é igualmente indispensável reconhecer os métodos eficazes para tal. Manter a comunicação uniforme entre os profissionais quanto às orientações dos cuidados, considerar as necessidades de cada família, utilizar-se de ferramentas demonstrativas e material impresso para facilitar a compreensão da família e assegurar-se de que todo o conteúdo necessário foi exposto e compreendido para o cuidado seguro no domicílio são alguns desses métodos.

Recomenda-se, portanto, a sistematização das orientações para a transição do cuidado para o domicílio e ações educativas por meio da construção de um roteiro estruturado para padronização do processo e compartilhamento de responsabilidades entre a equipe multiprofissional sob a liderança do enfermeiro. Esta iniciativa corrobora para as melhorias necessárias neste processo de cuidado e agregaria valor ao trabalho multiprofissional com implicações direta as crianças e famílias.

Podem-se destacar como limitações deste estudo a coleta de dados realizada em um único local de pesquisa, o que restringe o número de participantes de cada área profissional, limitando a generalização dos dados, bem como a não inclusão dos técnicos de enfermagem como participantes do estudo, já 
que estes permanecem muito próximos a criança e a família por mais tempo durante o período da internação hospitalar, o que favorece a identificar as necessidades da família nos cuidados.

A escassez de outros estudos na literatura nacional consultada com a mesma temática salienta a relevância deste estudo e aponta a necessidade de novas pesquisas com o mesmo objeto de estudo. Sugere-se também a realização de novos estudos com a participação de familiares de crianças já em tratamento, o que poderia enriquecer a identificação de informações elementares a partir de dificuldades que enfrentaram após a primeira alta hospitalar.

\section{CONTRIBUIÇÕES DOS AUTORES}

Desenho do estudo. Sara Raquel Kuntz. Wiliam Wegner. Coleta ou produção dos dados. Sara Raquel Kuntz. Wiliam Wegner.

Análise de dados. Sara Raquel Kuntz. Wiliam Wegner. Luiza Maria Gerhardt. Anali Martegani Ferreira. Maitê Telles dos Santos. Maria Cristina Flurin Ludwig.

Interpretação dos resultados. Sara Raquel Kuntz. Wiliam Wegner. Luiza Maria Gerhardt. Anali Martegani Ferreira. Maitê Telles dos Santos. Maria Cristina Flurin Ludwig.

Redação e revisão crítica do manuscrito. Sara Raquel Kuntz. Luiza Maria Gerhardt. Anali Martegani Ferreira. Maitê Telles dos Santos. Maria Cristina Flurin Ludwig. Wiliam Wegner.

Aprovação da versão final do artigo. Sara Raquel Kuntz. Luiza Maria Gerhardt. Anali Martegani Ferreira. MaitêTelles dos Santos. Maria Cristina Flurin Ludwig. Wiliam Wegner.

Responsabilidade por todos os aspectos do conteúdo e a integridade do artigo publicado. Sara Raquel Kuntz. Luiza Maria Gerhardt. Anali Martegani Ferreira. Maitê Telles dos Santos. Maria Cristina Flurin Ludwig. Wiliam Wegner.

\section{EDITOR ASSOCIADO}

\author{
Aline Cristiane Okido
}

\section{REFERÊNCIAS}

1. Cruz EF, Silva LF, Goes FGB, Aguiar RCB, Moraes JRMM. Orientações de enfermagem junto à criança em tratamento quimioterápico antineoplásico. Rev. Eletr. Enf. Goiânia. 2014 abr/jun;16(2):378-85. http://dx.doi.org/10.5216/ree.v16i2.27009.

2. Aburn G, Gott M. Education given to parents of children newly diagnosed with acute lymphoblastic leukemia: the parent's perspective. Peditr. Nurs. 2014 set;40(5):243-56. PMID: 25929116.

3. Silva-Rodrigues FM, Bernardo CSG, Alvarenga WA, Janzen DC, Nascimento LC. Transição de cuidados para o domicílio na perspectiva de pais de filhos com leucemia. Rev Gaúcha Enferm. 2019;40:e20180238. http://dx.doi.org/10.1590/1983-1447.2019.20180238. PMid:31188974.

4. World Health Organization. Transitions of care: technical series on safer primary care [Internet]. Geneva:WHO; 2016 [citado 2019 nov 19]. Disponível em: https://apps.who.int , bitstream , 9789241511599-eng

5. Landier W, Ahern J, Barakat LP, Bhatia S, Bingen KM, Bondurant PG et al. Patient/Family Education for Newly Diagnosed Pediatric Oncology Patients: consensus recommendations from a Children's Oncology Group Expert Panel. J Pediatr Oncol Nurs. 2016;33(6):422-31. http:// dx.doi.org/10.1177/1043454216655983. PMid:27385664.
6. Rodgers CC, Laing CM, Herring RA, Tena N, Leonardelli A, Hockenberry $\mathrm{M}$ et al. Understanding Effective Delivery of Patient and Family Education in Pediatric Oncology: a systematic review from the Children's Oncology Group. J Pediatr Oncol Nurs. 2016;33(6):432-46. http://dx.doi. org/10.1177/1043454216659449. PMid:27450361.

7. Landier W, Leonard M, Ruccione KS. Children's Oncology Group's 2013 Blueprint for Research: nursing discipline. Pediatr Blood Cancer. 2013;60(6):1031-6. http://dx.doi.org/10.1002/pbc.24415. PMid:23255369.

8. Polit DF, Beck CT. Fundamentos de pesquisa em enfermagem: avaliação de evidências para a prática da enfermagem. $7^{\underline{a}}$ ed. Porto Alegre: Artmed; 2011.

9. Minayo MC. (Org.). Pesquisa social, teoria, método e criatividade. 29a ed. Petrópolis (RJ): Vozes; 2010.

10. Resolução n. 466, de 12 de dezembro de 2012 (BR). Diretrizes e normas regulamentadoras de pesquisa em seres humanos. Diário Oficial da União, Brasília (DF), 2012.

11. Nair M, Paul LT, Latha PT, Parukkutty K. Parents' knowledge and attitude regarding their child's cancer and effectiveness of initial disease counseling in pediatric oncology patients. Indian J Palliat Care. 2017;23(4):393-8. http://dx.doi.org/10.4103/IJPC.IJPC_83_17. PMid:29123344.

12. Nemati S, Rassouli M, llkhani M, Baghestani AR. Perceptions of family caregivers of cancer patients about the challenges of caregiving: a qualitative study. Scand J Caring Sci. 2018;32(1):309-16. PMid:28869659.

13. Delatorre PG, Sá SPC, Valente GSC, Silvino ZR. Planning for hospital discharge as a strategy for nursing care: integrative review. J Nurs UFPE on line. 2013:7(esp):7151-9. http://dx.doi.org/10.5205/reuol.476742136-1-ED.0712esp201324.

14. Zatoni DCP, Lacerda MR, Hermann AP, Gomes IM, Nascimento JD, Rodrigues JAP. Suggestions for the improvement of guidance at the hospital discharge of children in post hematopoietic stem cell transplantation. Cogitare Enfermagem. 2017;22(4):e50265. http://dx.doi.org/10.5380/ ce.v22i4.50265.

15. Wilson Smith MG, Sachse K, Perry MT. Road to Home Program: a performance improvement initiative to increase family and nurse satisfaction with the discharge education process for newly diagnosed pediatric oncology patients. J Pediatr Oncol Nurs. 2018;35(5):368-74. http://dx.doi.org/10.1177/1043454218767872. PMid:29633659.

16. Silva KL, Sena RR, Castro WS. A desospitalização em um hospital público geral de Minas Gerais: desafios e possibilidades. Rev Gaúcha Enferm. 2018;38(4):e67762. http://dx.doi.org/10.1590/1983-1447.2017.04.67762. PMid:29933425.

17. Withycombe JS, Andam-Mejia R, Dwyer A, Slaven A, Windt K, Landier W. A Comprehensive Survey of Institutional Patient/Family Educational Practices for Newly Diagnosed Pediatric Oncology Patients. J Pediatr Oncol Nurs. 2016;33(6):414-21. http://dx.doi.org/10.1177/1043454216652857. PMid:27283721.

18. Monnerat CP, Silva LF, Souza DK, Aguiar RCB, Curcino EG, Pacheco STA. Health education strategy with family members of children in continuous medication. J Nurs UFPE on line. 2016;10(11):3814-22. https://doi.org/10.5205/reuol.9881-87554-1-EDSM1011201601.

19. Lima MFS, Minetto RC. Conhecimento de pacientes onco-hematológicos em tratamento quimioterápico sobre os cuidados para prevenção de infecções [Internet]. Com. Ciênc Saúde (Porto Alegre). 2014; [citado 2019 nov 19];25(1):35-44. Disponível em: https://bvsms.saude.gov.br/ bvs/periodicos/conhecimento_pacientes_onco.pdf

20. Klassmann J, Kochia KRA, Furukawa TS, Higarashi IH, Marcon SS. Experiência de mães de crianças com leucemia: sentimentos acerca do cuidado domiciliar. Rev Esc Enferm USP. 2008;42(2):321-30. http:// dx.doi.org/10.1590/S0080-62342008000200016. PMid:18642745.

21. The Paediatric Society of New Zealand and Starship Foundation. Checklist before leaving hospital [Internet]. Paraparaumu, NZ: The Paediatric Society of New Zealand; 2018 [citado 2019 nov 19]. Disponível em: https://www.kidshealth.org.nz/checklist-leaving-hospital?language=zhhans

22. Rodgers C, Bertini V, Conway MA, Crosty A, Filice A, Herring RA et al. A Standardized Education Checklist for Parents of children newly diagnosed with cancer: a report from the Children's Oncology Group. J Pediatr Oncol Nurs. 2018 jul;35(4):235-46. http://dx.doi.org/10.1177/1043454218764889. PMid:29589818. 